\title{
PD-L1 tumour expression is predictive of pazopanib response in soft tissue sarcoma
}

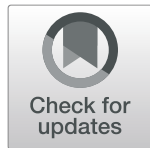

Sang Kyum Kim ${ }^{1 \dagger}$, Jee Hung Kim²†, Seung Hyun Kim³, Young Han Lee ${ }^{4}$, Jung Woo Han ${ }^{5}$, Wooyeol Baek Ha Young Woo ${ }^{1}$, Min Kyung Jeon ${ }^{7}$ and Hyo Song Kim ${ }^{7^{*}}$ (D)

\begin{abstract}
Background: Pazopanib, a multitargeted tyrosine kinase inhibitor, is recommended as the standard treatment for refractory soft tissue sarcoma (STS). However, there are comparatively few molecular determinants for predicting pazopanib efficacy. Based on correlative studies regarding the predictive impact of PD-L1, we investigated the clinical relevance of PD-L1 expression and evaluated its value for predicting pazopanib efficacy.

Methods: Tumour tissues from patients with advanced STS who went on to receive pazopanib were assessed for PD-L1 expression. Immunohistochemistry was performed using an anti-PD-L1 antibody, and the PD-L1 tumour proportion score (TPS) was calculated as the percentage of at least 100 viable cells with positive expression, defined as TPS $\geq 1 \%$.

Results: Among the 67 patients, 8 (11.9\%) achieved partial response and a median progression-free survival (PFS) of 4.8 months (95\% Cl 3.8-5.7). PD-L1 expression in tumour cells was detected in 13 (19.4\%) cases and the TPS scores ranged from 1 to 100\%, as follows: $0(n=54,80.6 \%), 1-9 \%(n=3,4.5 \%), 10-49 \%(n=9,13.4 \%)$, and $\geq 50 \%(n=1$, 1.5\%). PD-L1 positive tumours exhibited a poorer response to pazopanib treatment than the PD-L1 negative tumours ( $\%$ vs $14.8 \%, P=0.07$ ). PD-L1-positive tumours had significantly shorter PFS than the PD-L1-negative tumours (median PFS 2.8 vs 5.1 months, $P=0.003$ ), and PD-L1 positivity was an independent predictor of poor response to pazopanib treatment (HR 2.77, 95\% Cl; $1.45-5.56, P=0.006)$.

Conclusion: We identified that PD-L1 expression can help predict the clinical outcome of patients with advanced STS treated with pazopanib. Based on our study, stratification should be actively considered in order to identify patients who will benefit from pazopanib or further therapeutic strategies for future clinical trials.
\end{abstract}

Keywords: Soft tissue sarcoma, Pazopanib, PD-L1, Biomarker

\section{Background}

Soft tissue sarcomas (STSs) comprise a rare and heterogeneous group of tumours that originate from mesenchymal cells and account for $1 \%$ of all adult malignancies [1]. Although the primary treatment is usually surgery and/or radiotherapy, up to $40 \%$ of the patients experience tumour recurrence, and the

\footnotetext{
* Correspondence: hyosong77@yuhs.ac

†Sang Kyum Kim and Jee Hung Kim contributed equally to this work.

${ }^{7}$ Division of Medical Oncology, Department of Internal Medicine, Yonsei Cancer Center, Yonsei University College of Medicine, Seoul, South Korea Full list of author information is available at the end of the article
}

survival rate is poor, with a median survival overall of 12 months [2].

For patients with advanced STS, palliative chemotherapy generally takes the form of doxorubicin- or ifosfamide-based regimens [2, 3]. If first-line treatment fails, trabectedine, dacarbazine and gemcitabine, and/or docetaxel, have been approved as salvage treatments. Pazopanib is a multitargeted tyrosine kinase inhibitor that shows activity against vascular endothelial growth factor, platelet-derived growth factor, fibroblast growth

(c) The Author(s). 2021 Open Access This article is licensed under a Creative Commons Attribution 4.0 International License, which permits use, sharing, adaptation, distribution and reproduction in any medium or format, as long as you give appropriate credit to the original author(s) and the source, provide a link to the Creative Commons licence, and indicate if changes were made. The images or other third party material in this article are included in the article's Creative Commons licence, unless indicated otherwise in a credit line to the material. If material is not included in the article's Creative Commons licence and your intended use is not permitted by statutory regulation or exceeds the permitted use, you will need to obtain permission directly from the copyright holder. To view a copy of this licence, visit http://creativecommons.org/licenses/by/4.0/ The Creative Commons Public Domain Dedication waiver (http://creativecommons.org/publicdomain/zero/1.0/) applies to the data made available in this article, unless otherwise stated in a credit line to the data. 
factor receptors, and c-kit. In a stratified phase II trial for advanced STS [4], pazopanib displayed anti-cancer activity against leiomyosarcoma and synovial sarcoma, but not liposarcoma. In a subsequent phase III Pazopanib for Metastatic Soft-Tissue Sarcoma (PALETTE) trial designed for investigating the effect of pazopanib on non-adipocytic STS, it was observed that pazopanib treatment resulted in an improved progression-free survival (3 months) compared to that observed in response to the placebo [5]. While pazopanib is currently recommended as a standard treatment, patient response to this drug is still modest and the improvement of overall survival is not significant. We hypothesise that identifying molecular predictors to select patients who might benefit from pazopanib treatment might improve the efficacy of this drug. While previous studies have reported a number of clinical parameters as predictors of pazopanib efficacy, including circulating angiogenic factors and neutrophil to lymphocyte ratios, the application of these parameters has been limited thus far [6,7].

Based on our understanding of the mechanisms by which tumours evade the immune response, immune checkpoint inhibitor-based therapies (e.g.: pembrolizumab monotherapy, combinatorial therapy with nivolumab and ipilimumab, a cytotoxic T-lymphocyteassociated antigen 4 (CTLA-4) inhibitor) have been developed which show an effect on advanced STS $[8,9]$. Programmed death-ligand 1 (PD-L1) is aberrantly expressed in several subtypes of STS and has been associated with poor prognosis and adverse features $[10,11]$. Recently, a correlation between PD-L1 expression and clinical outcome was reported in renal cell carcinoma (RCC). Choueiri et al. showed that patients with higher PD-L1 expression had worse overall survival when treated with pazopanib or sunitinib [12]. However, the predictive impact of PD-L1 in STS remains unclear, and further research is warranted to provide more detailed therapeutic guidance regarding the treatment of this disease.

In this study, we investigated PD-L1 expression in STS tissues and evaluated the clinical relevance of its expression in different STS subtypes. In addition, we also evaluated the correlation between the expression of PD-L1 and treatment outcomes of patients who received pazopanib, for facilitating the identification of a molecular biomarker that could aid the design of effective STS treatments.

\section{Methods}

\section{Patient and study procedure}

We retrospectively selected 91 patients who were pathologically diagnosed with STS, from September 2013 to December 2019, at Yonsei Cancer Center, Yonsei University College of Medicine. Among these patients, 24 were excluded due to the lack of available specimens $(n=13)$ or insufficient tumour content $(n=11)$; 67 patients were finally included in this study. To select the most representative formalin-fixed paraffin-embedded (FFPE) tissues for immunohistochemistry, samples were mounted on slides, stained with haematoxylin and eosin, and reviewed by two pathologists (SKK and HYW). These tissue used for analysis were before pazopanib treatment.

Clinical information, including age, sex, etiology, Eastern Cooperative Oncology Group (ECOG) performance status, French Federation of Cancer Centers Sarcoma Group (FNCLCC) system score, staging and previous treatment data, were extracted from the hospital records. The study was reviewed and approved by the Institutional Review Board of the Yonsei Cancer Center (IRB. 4-2017-1023) in accordance with the Declaration of Helsinki and the Guidelines for Good Clinical Practice.

\section{Immunohistochemistry and evaluation of PD-L1 expression and tumour infiltrating lymphocytes (TIL) score}

In patients treated with pazopanib in combination with anti-PD-L1 blockade, PD-L1 expression was analysed using an anti-PD-L1 antibody (clone SP263, Ventana). From each block, $5 \mu \mathrm{m}$ sections were cut and stained using the anti-PD-L1 antibody on an automated staining platform (Benchmark ULTRA; Ventana). An OptiView DAB IHC Detection Kit and an OptiView Amplification Kit (both Ventana) were used according to the manufacturer's recommendations for the visualisation of PD-L1 protein. The PD-L1 tumour proportion score (TPS) was expressed as the percentage of at least 100 viable cells exhibiting complete or partial membrane staining and a three-tiered system was then applied using the following thresholds: $<1 \%, 1-49 \%$ and $\geq 50 \%$. Positive expression was defined as TPS $\geq 1 \%$. Macrophages were used as an internal control in order to validate the adequacy of the PD-L1 staining.

The percentage of intratumoural and stromal tumour infiltrating lymphocytes (TILs) was evaluated using the criteria described and published by the International Immuno-Oncology Biomarker Working Group [13]. Briefly, all mononuclear cells, excluding neutrophils, were scored, and the average number of TILs in the tumour area was assessed as a continuous variable. TIL scores were classified into the following three groups: negative $(<1 \%)$, low $(1-9 \%)$, intermediate $(10-59 \%)$, and high $(\geq 60 \%)$, by adopting the definition published by Ogiya et al. [14]

\section{Statistical analysis}

Progression-free survival (PFS) was defined as the time from the start of pazopanib treatment until the date of 
disease progression or death resulting from any cause. Overall survival (OS) was measured from the start of pazopanib treatment with advanced STS to the date of death due to any cause. Survival difference was analysed using the Kaplan-Meier method and was assessed using the log-rank test. Overall response rate (ORR) was calculated as the percentage of patients experiencing a confirmed complete response (CR) or partial response (PR), as per the Response Evaluation Criteria in Solid Tumors (RECIST) 1.1 guidelines. The associations between clinical features and each of the dichotomised groups were analysed using the chi-square test or Fisher's exact test. All $P$-values were two-sided and $p<0.05$ was considered significant. Statistical analysis was conducted using SPSS (version 21.0; SPSS, Inc., Chicago, IL) and GraphPad Prism version 5 (Graph Pad Software Inc., San Diego, CA, USA.

\section{Results}

\section{Clinicopathological characteristics of sarcoma patients} FFPE primary tumour specimens from a total of 67 patients with unresectable or metastatic STS were analysed for PD-L1 expression using immunohistochemistry. Fifty-two cases $(77.6 \%)$ were made from surgical specimens and 15 (22.4\%) from biopsy samples. And 58 cases $(86.6 \%)$ were from the primary tumor site and 9 (13.4\%) from metastasis sites. Twelve cases (17.9\%) were tissue samples obtained after chemotherapy, of which 7 cases were after the first chemotherapy and 5 cases were after the second chemotherapy or more. The most commonly observed histologies were leiomyosarcoma $(n=18)$, undifferentiated pleomorphic sarcoma (UPS; $n=13$ ), angiosarcoma $(n=8)$, and synovial sarcoma $(n=6)$. The majority of the tumours were high grade (FNCLCC grade 2 or $3 ; 91.0 \%$ ) and $61.2 \%$ of them arose in the abdomen or thorax. All patients had received at least one previous regimen of chemotherapy, mainly doxorubicin or gemcitabine plus docetaxel based (Table 1).

\section{Correlation between PD-L1 expression and the TIL score} Overall, PD-L1 expression in the tumour cells was detected in $13(19.4 \%)$ of the 67 patients, with TPS scores ranging from 1 to $100 \%$ positive staining in the following manner: 0 ( $n=54,80.6 \%), 1-9 \%(n=3,4.5 \%), 10-49 \%$ $(n=9,13.4 \%)$, and $\geq 50 \%(n=1,1.5 \%)$ positive staining. Representative images are provided in Fig. 1. PD-L1positive $(+)$ cases were common in UPS, angiosarcoma, malignant peripheral nerve sheath tumour (MPNST), and leiomyosarcoma but none of the synovial sarcoma tumours exhibited PD-L1 expression. PD-L1 (+) patients tended to present a higher grade and head/neck primary tumour.

The inflammatory response, as represented by the presence of TILs, was simultaneously evaluated in the following manner: negative $(n=39,58.2 \%)$, low TIL ( $n=$ $16,23.9 \%)$, intermediate TIL $(n=11,16.4 \%)$, and high TIL ( $\mathrm{n}=1,1.5 \%)$ sarcoma. There was a possible correlation between PD-L1-positive staining and TILs, and the percentage of TILs showed a tendency to increase according to the degree of PD-L1-positive staining $(P=$ 0.09 , Table 2). No significant difference was detected when the TIL score was evaluated with respect to other clinical characteristics (age, sex, ECOG performance, histologic subtype, and primary tumour site).

\section{Correlation between PD-L1 expression with pazopanib efficacy}

Of the 67 patients evaluated, 8 achieved a PR, 36 had stable disease, and 23 exhibited progressive disease phenotypes, resulting in an ORR of $11.9 \%$ (Fig. 2a). Responses were first detected at a median of 2.3 months (range 1.8-8.2) after treatment initiation and lasted for a median of 6.5 months (range 2.1-19.6). Responder who showed PR had higher PD-L1 negativity than nonresponder who showed $\mathrm{SD}$ and progression disease (PD) (100\% versus $83.3 \%$ versus $69.6 \%$, Fig. $2 \mathrm{~b}$ ). The ORRs of the different histological subtypes were as follows; 2 PRs (11.1\%) among 18 leiomyosarcomas, 1 (7.7\%) in 13 UPS, $1(12.5 \%)$ of 8 angiosarcomas, and 1 (33.3\%) of 3 epithelioid sarcomas. In synovial sarcoma, 3 PRs (50\%) were detected, of which 2 were biphasic and 1 was monophasic (Fig. 2c). Brief case descriptions of PD-L1 (+) expression are supplied in Supplementary Table 1.

Fifty-nine patients $(88.1 \%)$ exhibited progressive events and the median PFS was 4.8 months (95\% CI 3.8-5.7). Patients with PD-L1 $(+)$ expression had significantly shorter PFS than PD-L1 (-) patients (median PFS 2.8 vs 5.1 months, $P=0.003$, Fig. 3a). This trend was consistent for second-line-treated patients (Fig. 3b, median 2.8 vs 5.0 months, $P<0.001)$. The median OS was 10.1 months $(95 \%$ CI 5.42-14.78), and PD-L1-negative patients had a slightly better OS than PD-L1-positive patients, although this was not significant (12.6 vs 7.9 months, $P=0.11$, Fig. $3 c$ ).

In multivariate Cox regression analyses, only PD-L1 (+) expression was identified to be an independent factor for determining poor PFS upon pazopanib treatment (HR 2.77, 95\% CI; 1.45-5.56, $P=0.006$, Table 3). Further analyses using TILs showed that a combination of PDL1 (+) and higher TIL expression counts correlated with shorter PFS, and that patients positive for both PD-L1 and TILs had the shortest PFS [5.1 vs 4.3 vs 2.7 months for both PD-L1 (-) and TIL (-) vs PD-L1 (+) or TIL (+) vs both PD-L1 (+) and TIL (+), P=0.035, Fig. 3d].

\section{Discussion}

Using a standardised assay, in the current study we report the expression of PD-L1 in pre-treated STS tumour tissues. Furthermore, our study unravels the value of 
Table 1 Patient characteristics.

\begin{tabular}{|c|c|c|c|c|}
\hline Variables & Total & PD-L1 (-) & PD-L1 (+) & $P$ value \\
\hline & & $54(80.6 \%)$ & $13(19.4 \%)$ & \\
\hline Age (Median) & 48 & $51(38-73)$ & $45(22-72)$ & \\
\hline Sex & & & & 0.82 \\
\hline Male & 38 & $31(81.6 \%)$ & $7(18.4 \%)$ & \\
\hline Female & 29 & $23(79.3 \%)$ & $6(20.7 \%)$ & \\
\hline ECOG & & & & 0.99 \\
\hline 0 & 3 & $3(100 \%)$ & $0(0.0 \%)$ & \\
\hline 1 & 36 & $28(77.8 \%)$ & $8(22.2 \%)$ & \\
\hline 2 & 26 & $21(80.8 \%)$ & $5(19.2 \%)$ & \\
\hline Not available & 2 & $2(100 \%)$ & $0(0.0 \%)$ & \\
\hline Histologic variant & & & & 0.22 \\
\hline Leiomyosarcoma & 18 & $15(83.3 \%)$ & $3(16.7 \%)$ & \\
\hline Undifferentiated pleomorphic sarcoma & 13 & $9(69.2 \%)$ & $4(30.8 \%)$ & \\
\hline Angiosarcoma & 8 & $5(62.5 \%)$ & $3(37.5 \%)$ & \\
\hline Synovial sarcoma & 6 & $6(100 \%)$ & $0(0.0 \%)$ & \\
\hline Myofibroblastic sarcoma & 6 & $5(83.3 \%)$ & $1(16.7 \%)$ & \\
\hline MPNST & 5 & $3(60 \%)$ & $2(40 \%)$ & \\
\hline $\mathrm{Etc}^{*}$ & 11 & $11(100 \%)$ & $0(0.0 \%)$ & \\
\hline Primary site & & & & 0.23 \\
\hline Abdomen/pelvis & 23 & $20(87.0 \%)$ & $3(13.0 \%)$ & \\
\hline Extremity & 22 & 19 (86.4\%) & $3(13.6 \%)$ & \\
\hline Thorax & 18 & $13(72.2 \%)$ & $5(27.8 \%)$ & \\
\hline Head/neck & 4 & $2(50 \%)$ & $2(50 \%)$ & \\
\hline FNCLCC grade & & & & 0.36 \\
\hline । & 6 & $6(100 \%)$ & $0(0.0 \%)$ & \\
\hline$\|$ & 33 & $27(81.8 \%)$ & $6(19.2 \%)$ & \\
\hline III & 28 & $21(75 \%)$ & $7(25 \%)$ & \\
\hline Number of previous chemotherapy & & & & 0.83 \\
\hline 1 & 48 & $38(79.2 \%)$ & $10(20.8 \%)$ & \\
\hline 2 & 18 & $15(83.3 \%)$ & $3(16.7 \%)$ & \\
\hline 3 & 1 & $1(100 \%)$ & $0(0.0 \%)$ & \\
\hline Type of previous chemotherapy received & & & & NA \\
\hline Doxorubicin monotherapy & 9 & $7(77.8 \%)$ & $2(22.2 \%)$ & \\
\hline Ifosfamide monotherapy & 3 & $2(66.7 \%)$ & $1(33.3 \%)$ & \\
\hline Paclitaxel & 4 & $3(75.0 \%)$ & $1(25.0 \%)$ & \\
\hline Doxorubicin combination & 33 & $28(84.8 \%)$ & $5(15.2 \%)$ & \\
\hline Ifosfamide combination & 4 & $3(66.7 \%)$ & $1(33.3 \%)$ & \\
\hline Cyclophosphamide based & 14 & $11(78.6 \%)$ & $3(21.4 \%)$ & \\
\hline Gemcitabine/docetaxel & 14 & $12(85.7 \%)$ & $2(14.3 \%)$ & \\
\hline
\end{tabular}

* 8th edition of the American Joint Committee on Cancer guideline of tumor, node, and metastasis (TNM) classification

*Etc: ASPS (alveolar soft part sarcoma, $n=3$ ), epithelioid sarcoma $(n=3)$, DSRCT (desmoplastic small round cell tumors, $n=1$ ), osteosarcoma $(n=1)$, liposarcoma $(n=1)$, rhabdomyosarcoma $(n=1)$, and PECOMA (perivascular epithelioid cell tumors, $n=1$ )

Abbreviation: Eastern Cooperative Oncology Group (ECOG), malignant peripheral nerve sheath tumor (MPNST), Fédération Nationale des Centres de Lutte Contre le Cancer (FNCLCC) 

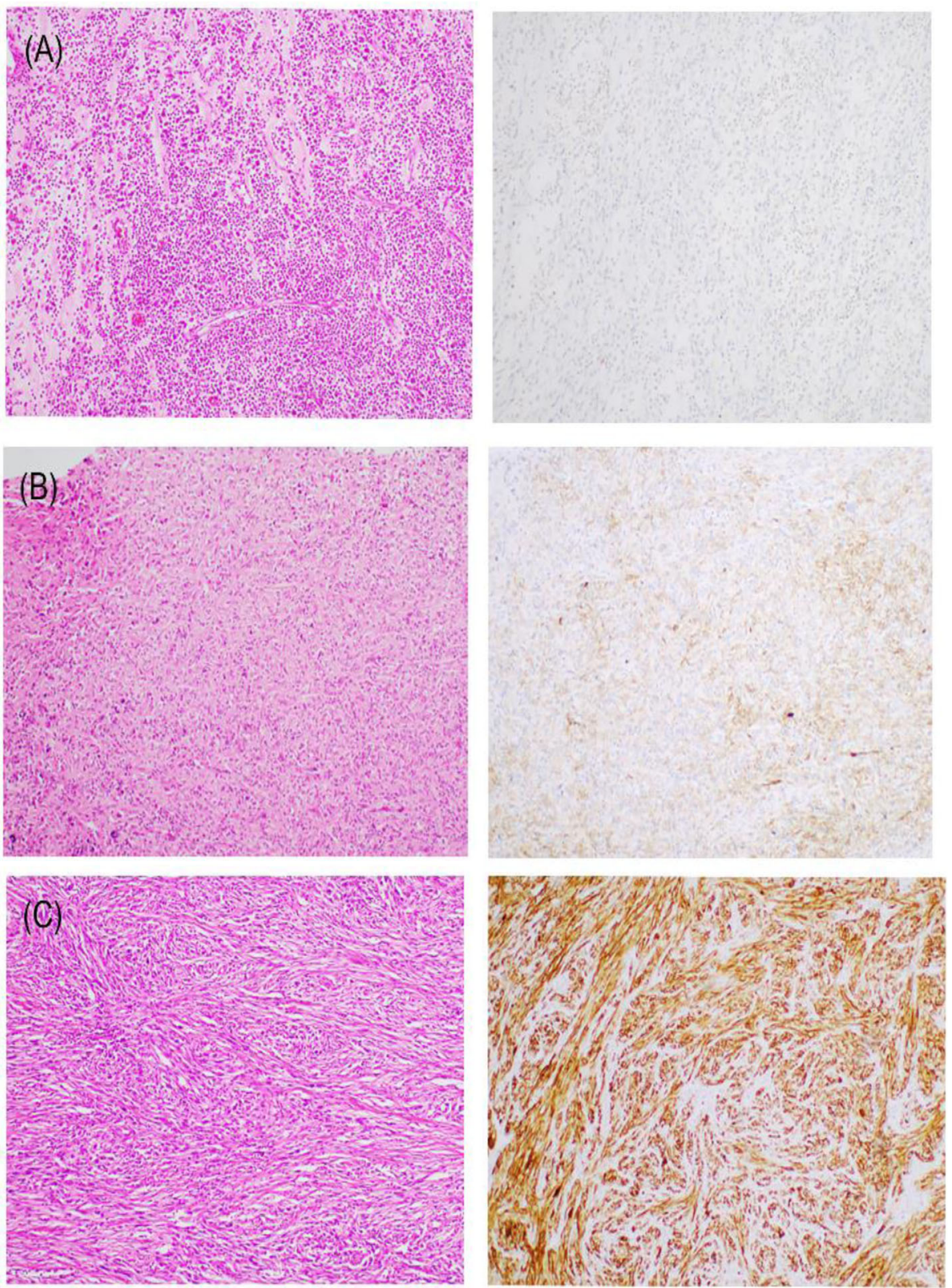

Fig. 1 Immunohistochemistry for PD-L1 expression in STS. Representative images of PD-L1-negative $(\mathbf{a}$, TPS 0\%, $\times 100)$ and -positive cases (b, TPS 40\%, $\times 100)(\mathbf{c}$, TPS 100\%, × 100). Abbreviation: Programmed death-ligand 1(PD-L1), soft tissue sarcoma (STS), tumour proportion score (TPS)

Table 2 Correlation between PD-L1 expression and TIL

\begin{tabular}{|c|c|c|c|c|c|c|}
\hline & & PD-L1 (\%) & & & & Total \\
\hline & & 0 & $1-9$ & $10-49$ & $\geq 50$ & $P=0.09$ \\
\hline \multirow[t]{5}{*}{ TIL } & Negative (0) & 34 (62.9\%) & $1(33.3 \%)$ & $4(44.4 \%)$ & 0 & $39(58.2 \%)$ \\
\hline & Low (1-9) & $13(24.1 \%)$ & $2(66.7 \%)$ & $1(11.1 \%)$ & 0 & $16(23.9 \%)$ \\
\hline & Intermediate (10-59) & $6(11.1 \%)$ & 0 & $4(44.4 \%)$ & $1(100 \%)$ & $11(16.4 \%)$ \\
\hline & High $(\geq 60)$ & $1(1.9 \%)$ & 0 & 0 & 0 & $1(1.5 \%)$ \\
\hline & & $54(80.6 \%)$ & $3(4.5 \%)$ & $9(13.4 \%)$ & $1(1.5 \%)$ & \\
\hline
\end{tabular}



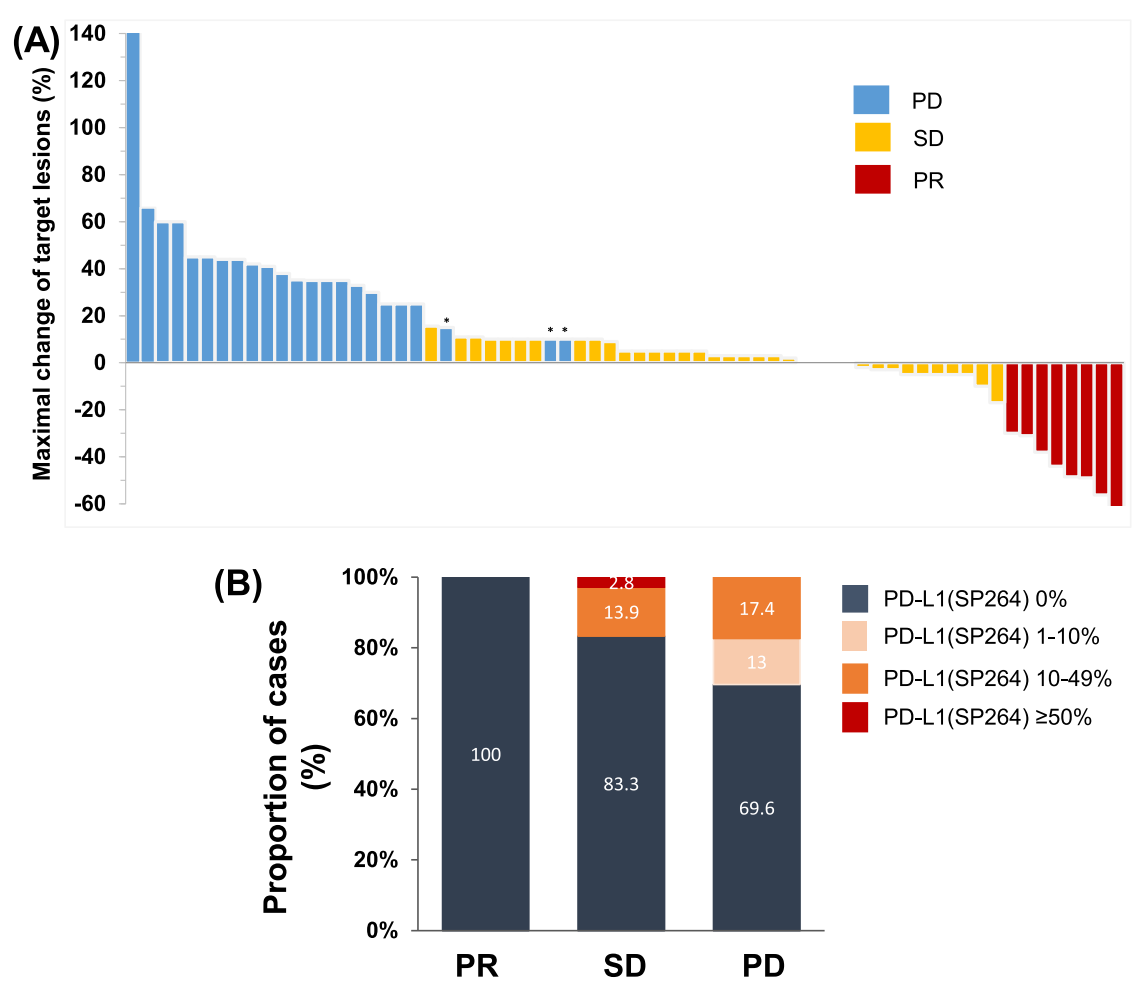

(C)

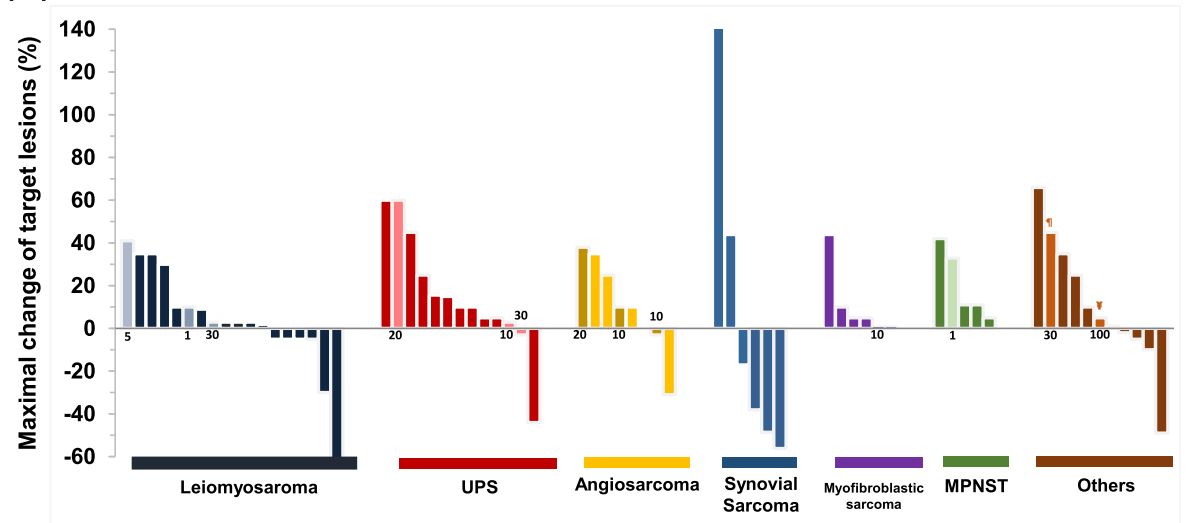

Fig. 2 Maximum response to pazopanib in patients with STS. (a) The waterfall plot represents percentage of maximum tumour reduction in response to treatment, assessed according to RECIST 1.1 criteria. * indicates newly developed lesions per RECIST 1.1. (b) Prevalence of PD-L1 expression in responders (PR) and non-responders (SD, PD), assessed according to RECIST 1.1 criteria. (c) Maximum tumour reduction according to histological subtypes and PD-L1 status. PD-L1 TPS score ( $\geq 1 \%)$ has been plotted on the X-axis and PD-L1 positive marked in light color bars. Indicates rhabdomyosarcoma and $¥$ indicates ASPS (Alveolar soft part sarcoma). Abbreviation: Soft tissue sarcoma (STS), Response Evaluation Criteria in Solid Tumors (RECIST) 1.1, Programmed death-ligand 1(PD-L1), tumour proportion score (TPS), PR (partial response), SD (stable disease), PD (progression disease). *This figure was generated with Microsoft Excel

PD-L1 for predicting pazopanib efficacy. To our knowledge, this is the first study to evaluate the role of PD-L1 expression in pazopanib response in STS.

Pazopanib, an inhibitor of angiogenesis and tumour cell proliferation, has been recognized as a salvage treatment for STS [5]. The approval of this drug was based on the results of the randomised phase III PALETTE trial, which showed that pazopanib improved PFS in patients with previously treated STS compared to placebo treatment. However, despite the prolonged PFS, no significant difference in OS was observed, and our understanding of the factors mediating pazopanib sensitivity and resistance is still poor. There is an urgent need for the identification of predictive biomarkers which can be used to select subgroups of patients who will benefit from pazopanib treatment. In the PALETTE trial, it was 

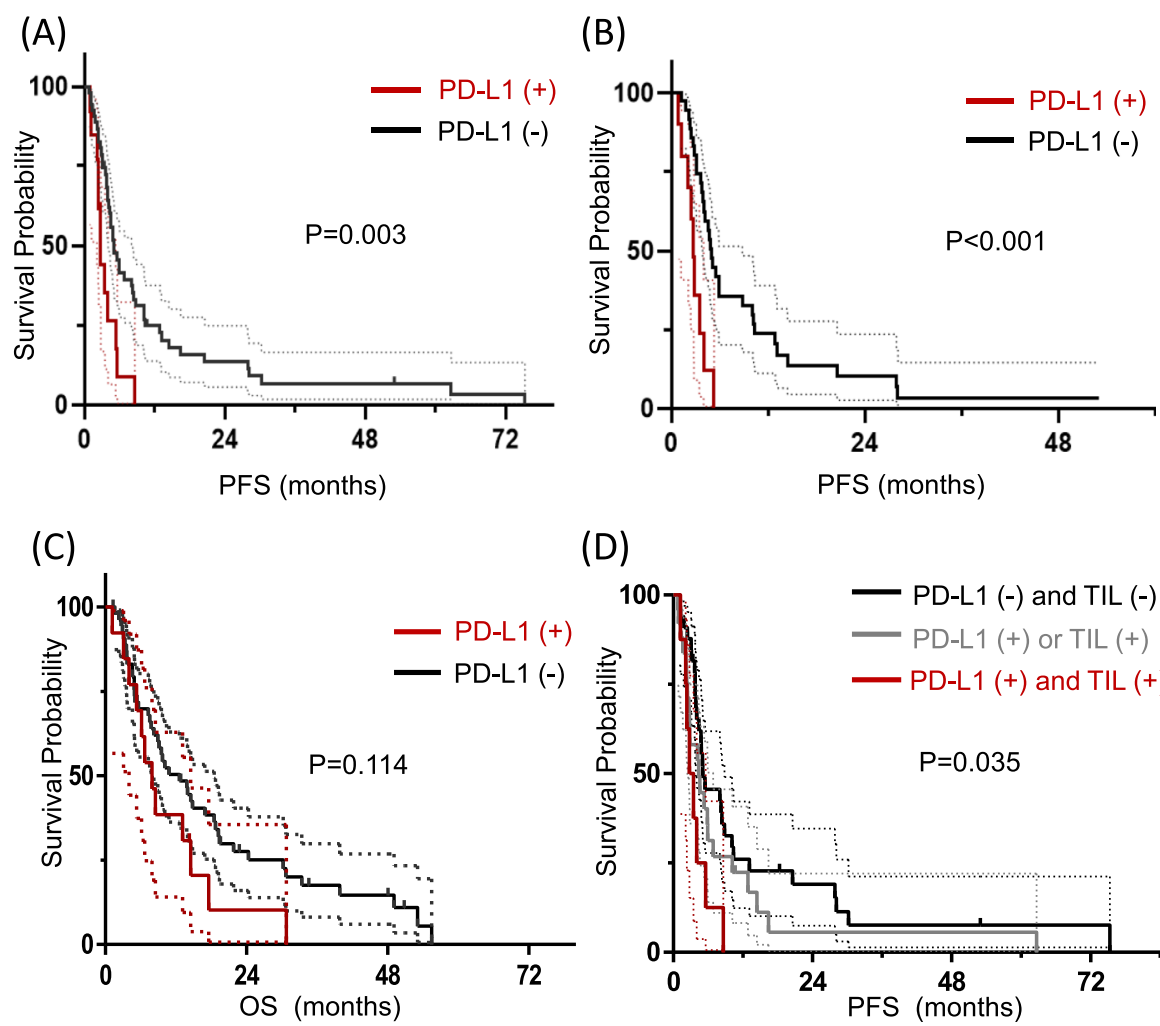

(D)

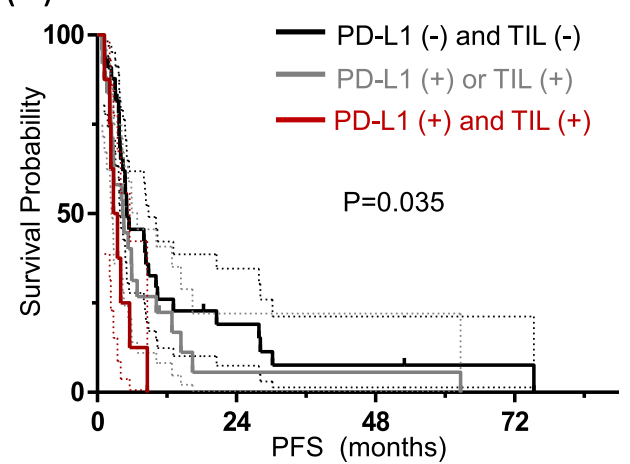

Fig. 3 Survival analyses based on PD-L1 expression. Kaplan-Meier survival curves for PFS for all patients (a) and those with second-line treatment (b). Kaplan-Meier survival curves for OS (c). PFS difference in response to the combined expression of PD-L1 and TILs (d). Abbreviation: Programmed death-ligand 1(PD-L1), progression-free survival (PFS), overall survival (OS), tumour infiltrating lymphocytes (TILS). *This figure was generated with GraphPad Prism version 5 (Graph Pad Software Inc., San Diego, CA, USA)

Table 3 Prognostic Factors for Progression-free survival

\begin{tabular}{lll}
\hline & Hazard Ratio (95\% Cl) & P value \\
\hline Performance (0 vs 1) & $0.74(0.39-1.44)$ & 0.38 \\
Number of previous chemotherapy (1 vs 2-3) & $0.67(0.29-1.57)$ & 0.36 \\
Sex (female vs male) & $1.27(0.65-2.49)$ & 0.49 \\
Age ( $\mathbf{4} \mathbf{8}$ years vs $>\mathbf{4 8}$ years) & $1.69(0.86-3.30)$ & 0.12 \\
ECOG (0-1 vs 2) & $0.74(0.39-1.44)$ & 0.38 \\
Grade (I vs II-III) & $1.22(0.43-3.45)$ & 0.77 \\
Histologic subtype & $1.17(0.49-2.06)$ & \\
(leiomyosarcoma and synovial sarcoma & & 0.95 \\
vs other subtypes $)$ & & 0.75 \\
Locoregional disease (yes vs no) & $1.10(0.60-2.03)$ & 0.65 \\
Liver metastases (yes vs no) & $0.75(0.22-2.58)$ & 0.006 \\
TIL (high vs low) & $2.77(1.45-5.56)$ & 0.197 \\
\hline
\end{tabular}

*other subtypes: undifferentiated pleomorphic sarcoma, angiosarcoma, myofibroblastic sarcoma, MPNST (malignant peripheral nerve sheath tumor), ASPS (Alveolar soft part sarcoma), epithelioid sarcoma, DSRCT, osteosarcoma, liposarcoma, rhabdomyosarcoma, and PECOMA

Abbreviation: Eastern Cooperative Oncology Group (ECOG), tumour infiltrating lymphocytes (TILs) 
noted that patients with leiomyosarcoma and synovial sarcoma exhibited better PFS upon pazopanib; however, a significant interaction with histological subtypes was not identified in predictive analysis. Other biological markers include post-treatment neutrophil-lymphocyte ratio, which was also suggested to be a robust predictive factor for pazopanib efficacy [7]. However, haematological parameters (such as neutrophil and lymphocyte counts) are unreliable because they can be easily influenced by other inflammatory conditions. Although a number of potential biomarkers have also been investigated, none of these are available as patient selection strategies for use in clinical practice.

Immune checkpoint inhibitors that block PD-1 and PD-L1 have exhibited remarkable efficacy with respect to the treatment of refractory solid tumours including melanoma and RCC, and substantial attention has been paid to assessing PD-L1 expression in STS. PD-L1 expression has been studied in various STS subtypes [10], and a recent meta-analysis also reported a role for PDL1 expression in the poor prognosis of bone and STS [15]. Interestingly, immunotherapy resulted in the generation of a response in certain sarcoma subtypes, mainly UPS, liposarcoma, and angiosarcoma $[8,9]$. The Cancer Genome Atlas (TCGA) analysis have described the following immune classification based on the composition of the tumor microenvironment in STS: immune-low, immune-high, and vascularized group. In this study, immune-high group (mainly UPS and liposarcoma) exhibited high expression of PD1, PDL2, and CTLA-4 [16]. Consistent with previous studies, we show that PD-L1 (+) was commonly observed in UPS, angiosarcoma, and leiomyosarcoma, and that PD-L1 (+) staining independently correlated with poor efficacy of pazopanib treatment. Furthermore, none of the synovial sarcoma tumours exhibited PD-L1 expression and this subtype exhibited a higher response to pazopanib treatment. Similarly, the synovial sarcoma subtype also showed favourable responses to pazopanib in the PALETTE trial [5]. Therefore, we propose that PD-L1 expression is associated with pazopanib resistance and that this hypothesis is worth further testing as a therapeutic strategy in future clinical trials.

A recent report described a correlation between PD-L1 expression on the tumour and the efficacy of pazopanib or sunitinib treatment in metastatic RCC. Patients whose tumours were TIL-positive and/or displayed high PD-L1 expression had poor survival outcomes in response to vascular endothelial growth factor (VEGF)-targeted agents [12]. A preclinical study showed that anti-PD-L1 therapy enhances tumour sensitivity to antiangiogenic therapy by inducing the development of endothelial venules that facilitate cytotoxic $\mathrm{T}$ cell activity and tumour lysis [17]. Therefore, on the basis that the VEGF receptor (VEGFR) plays a central role in immunosuppression, a strategy of combination treatments has been widely studied in RCC $[18,19]$. Similarly, the combination of a VEGF inhibitor, axitinib, with pembrolizumab was shown to have promising efficacy in advanced sarcomas, particularly in patients with advanced soft-part sarcoma [20]. Taken together, the combination of pazopanib and PD-L1 blockade may be a promising future therapeutic option for STS, and the final results of an ongoing trial (A Study of Pazopanib and Durvalumab for Metastatic Soft Tissue Sarcoma; ClinicalTrials.gov Identifier: NCT03798106) may help to confirm this premise.

To clarify the exact characteristics and prognostic role of PD-L1 expression, we carefully assessed a large cohort of patients with STS who were treated with pazopanib. Furthermore, from a practical perspective, three different PD-L1 antibodies (22C3, 28-8, and SP263) showed comparable analytical performance with respect to assessing PD-L1 expression [21]. Therefore, we used a companion diagnostic marker for approved anti-PD-L1 antibodies, SP263 PD-L1, in accordance with a standard protocol (instrument platform, staining procedure, and scoring methods) to provide a practical guideline. However, because of the retrospective nature of this study, future studies should evaluate a larger, prospective cohort to address the question of whether PD-L1 expression can serve as a clinical prognostic marker for STS.

\section{Conclusions}

Our study described a predictive role for PD-L1 expression in assessing the clinical outcomes for patients with advanced STS treated with pazopanib. This study provides important information that can guide treatment choices to better tailor the efficacy of pazopanib, thereby leading to new therapeutic strategies for sarcoma.

\section{Abbreviations}

STS: Soft tissue sarcoma; TPS: tumour proportion score; CTLA-4: cytotoxic Tlymphocyte-associated antigen 4; PD-L1: programmed death-ligand 1; FFPE: formalin-fixed paraffin-embedded; ECOG: Eastern Cooperative Oncology Group; FNCLCC: French Federation of Cancer Centers Sarcoma Group; TILs: tumour infiltrating lymphocytes; PFS: progression-free survival; OS: overall survival; ORR: overall response rate; CR: complete response; PR: partial response; RECIST): Response Evaluation Criteria in Solid Tumors; UPS: 1.1 undifferentiated pleomorphic sarcoma; MPNST: malignant peripheral nerve sheath tumour; ASPS: alveolar soft part sarcoma; DSRCT: desmoplastic small round cell tumors; PECOMA: perivascular epithelioid cell tumor; TCGA: The Cancer Genome Atlas; VEGF: to vascular endothelial growth factor; VEGFR: VEGF receptor

\section{Supplementary Information}

The online version contains supplementary material available at https:/doi. org/10.1186/s12885-021-08069-z.

\section{Additional file 1.}

Acknowledgements

Not applicable. 


\section{Authors' contributions}

SKK and HSK have made substantial contributions to concept and design of the study. JHK, SHK, YHL carried out analysis and interpretation of data. JWH, HYW, and MKJ have been involved in drafting the manuscript. JHK and WB have been carried out critical revision of manuscript for important intellectual contents. All authors read and approved the final manuscript.

\section{Funding}

This work was supported by a National Research Foundation of Korea (NRF) grant funded by the Korean government (MSIT) (Grant No.

2018R1A2B6003707, Hyo Song Kim) and a Severance Hospital Research fund for Clinical excellence grant (SHRC, grant no. C $C^{-2019}-0015$, Hyo Song Kim). The funding bodies had no role in the design of the study, data collection, analysis, interpretation of data, and writing of the manuscript.

\section{Availability of data and materials}

The anonymized data used and/or analyzed during the current study are available from the corresponding author on reasonable request.

\section{Declarations}

Ethics approval and consent to participate

The study was reviewed and approved by the Institutional Review Board of the Yonsei Cancer Center (IRB. 4-2017-1023) in accordance with the Declaration of Helsinki and the Guidelines for Good Clinical Practice.

\section{Consent for publication}

No relevant identifiable patient data.

\section{Competing interests}

The authors report no conflict of interest.

\section{Author details}

${ }^{1}$ Department of Pathology, Yonsei University College of Medicine, Seoul, South Korea. ${ }^{2}$ Division of Medical Oncology, Department of Internal Medicine, Gangnam Severance Hospital, Yonsei University College of Medicine, Seoul, South Korea. ${ }^{3}$ Department of Orthopedic Surgery, Yonsei University College of Medicine, Seoul, South Korea. ${ }^{4}$ Department of Radiology, Yonsei University College of Medicine, Seoul, South Korea. ${ }^{5}$ Division of Pediatric Hemato-Oncology, Department of Pediatrics, Yonsei University College of Medicine, Seoul, South Korea. ${ }^{6}$ Department of Plastic Surgery, Yonsei University College of Medicine, Seoul, South Korea. ${ }^{7}$ Division of Medical Oncology, Department of Internal Medicine, Yonsei Cancer Center, Yonsei University College of Medicine, Seoul, South Korea.

Received: 10 September 2020 Accepted: 18 March 2021 Published online: 31 March 2021

\section{References}

1. Clark MA, Fisher C, Judson I, Thomas JM. Soft-tissue sarcomas in adults. N Engl J Med. 2005;353(7):701-11. https://doi.org/10.1056/NEJMra041866.

2. Judson I, Verweij J, Gelderblom H, Hartmann JT, Schoffski P, Blay JY, et al. Doxorubicin alone versus intensified doxorubicin plus ifosfamide for firstline treatment of advanced or metastatic soft-tissue sarcoma: a randomised controlled phase 3 trial. Lancet Oncol. 2014;15(4):415-23. https://doi.org/1 0.1016/S1470-2045(14)70063-4.

3. Hensley ML, Maki R, Venkatraman E, Geller G, Lovegren M, Aghajanian C, et al. Gemcitabine and docetaxel in patients with unresectable leiomyosarcoma: results of a phase II trial. J Clin Oncol. 2002;20(12):2824-31. https://doi.org/10.1200/JCO.2002.11.050.

4. Sleijfer S, Ray-Coquard I, Papai Z, Le Cesne A, Scurr M, Schoffski P, et al. Pazopanib, a multikinase angiogenesis inhibitor, in patients with relapsed or refractory advanced soft tissue sarcoma: a phase II study from the European organisation for research and treatment of cancer-soft tissue and bone sarcoma group (EORTC study 62043). J Clin Oncol. 2009;27(19):3126-32. https://doi.org/10.1200/JCO.2008.21.3223.

5. van der Graaf WT, Blay JY, Chawla SP, Kim DW, Bui-Nguyen B, Casali PG, et al. Pazopanib for metastatic soft-tissue sarcoma (PALETTE): a randomised, double-blind, placebo-controlled phase 3 trial. Lancet. 2012;379(9829):187986. https://doi.org/10.1016/S0140-6736(12)60651-5.
6. Sleijfer S, Gorlia T, Lamers C, Burger H, Blay JY, Le Cesne A, et al. Cytokine and angiogenic factors associated with efficacy and toxicity of pazopanib in advanced soft-tissue sarcoma: an EORTC-STBSG study. Br J Cancer. 2012; 107(4):639-45. https://doi.org/10.1038/bjc.2012.328.

7. Kobayashi H, Okuma T, Oka H, Hirai T, Ohki T, Ikegami M, et al. Neutrophilto-lymphocyte ratio after pazopanib treatment predicts response in patients with advanced soft-tissue sarcoma. Int J Clin Oncol. 2018;23(2):368-74. https://doi.org/10.1007/s10147-017-1199-6.

8. D'Angelo SP, Mahoney MR, Van Tine BA, Atkins J, Milhem MM, Jahagirdar BN et al. Nivolumab with or without ipilimumab treatment for metastatic sarcoma (Alliance A091401): two open-label, non-comparative, randomised, phase 2 trials. Lancet Oncol. 2018;19(3):416-26. https://doi.org/10.1016/S1470-2045(18)30006-8.

9. Tawbi HA, Burgess M, Bolejack V, Van Tine BA, Schuetze SM, Hu J, et al. Pembrolizumab in advanced soft-tissue sarcoma and bone sarcoma (SARC028): a multicentre, two-cohort, single-arm, open-label, phase 2 trial. Lancet Oncol. 2017; 18(11):1493-501. https://doi.org/10.1016/S1470-2045(17)30624-1.

10. Kim C, Kim EK, Jung H, Chon HJ, Han JW, Shin KH, et al. Prognostic implications of PD-L1 expression in patients with soft tissue sarcoma. BMC Cancer. 2016;16(1):434. https://doi.org/10.1186/s12885-016-2451-6.

11. Kim JR, Moon YJ, Kwon KS, Bae JS, Wagle S, Kim KM, et al. Tumor infiltrating PD1-positive lymphocytes and the expression of PD-L1 predict poor prognosis of soft tissue sarcomas. PLoS One. 2013;8(12):e82870. https://doi. org/10.1371/journal.pone.0082870.

12. Choueiri TK, Figueroa DJ, Fay AP, Signoretti S, Liu Y, Gagnon R, et al. Correlation of PD-L1 tumor expression and treatment outcomes in patients with renal cell carcinoma receiving sunitinib or pazopanib: results from COMPARZ, a randomized controlled trial. Clin Cancer Res. 2015;21(5):1071-7. https://doi.org/10.1158/1078-0432.CCR-14-1993.

13. Hendry S, Salgado R, Gevaert T, Russell PA, John T, Thapa B, et al. Assessing Tumor-Infiltrating Lymphocytes in Solid Tumors: A Practical Review for Pathologists and Proposal for a Standardized Method from the International Immuno-Oncology Biomarkers Working Group: Part 2: TILs in Melanoma, Gastrointestinal Tract Carcinomas, Non-Small Cell Lung Carcinoma and Mesothelioma, Endometrial and Ovarian Carcinomas, Squamous Cell Carcinoma of the Head and Neck, Genitourinary Carcinomas, and Primary Brain Tumors. Adv Anat Pathol. 2017;24(6):311-35.

14. Ogiya R, Niikura N, Kumaki N, Bianchini G, Kitano S, Iwamoto T, et al. Comparison of tumor-infiltrating lymphocytes between primary and metastatic tumors in breast cancer patients. Cancer Sci. 2016;107(12):1730-5. https://doi.org/10.1111/cas.13101.

15. Zheng C, You W, Wan P, Jiang X, Chen J, Zheng Y, et al. Clinicopathological and prognostic significance of PD-L1 expression in sarcoma: a systematic review and meta-analysis. Medicine (Baltimore). 2018;97(25):e11004. https:// doi.org/10.1097/MD.0000000000011004.

16. Petitprez F, de Reynies A, Keung EZ, Chen TW, Sun CM, Calderaro J, et al. B cells are associated with survival and immunotherapy response in sarcoma. Nature. 2020;577(7791):556-60. https://doi.org/10.1038/s41586-019-1906-8.

17. Allen E, Jabouille A, Rivera LB, Lodewijckx I, Missiaen R, Steri V, et al. Combined antiangiogenic and anti-PD-L1 therapy stimulates tumor immunity through HEV formation. Sci Transl Med. 2017:9(385).

18. McDermott DF, Huseni MA, Atkins MB, Motzer RJ, Rini Bl, Escudier B, et al. Clinical activity and molecular correlates of response to atezolizumab alone or in combination with bevacizumab versus sunitinib in renal cell carcinoma. Nat Med. 2018;24(6):749-57. https://doi.org/10.1038/s41591-018-0053-3.

19. Cella D, Grunwald V, Escudier B, Hammers HJ, George S, Nathan P, et al. Patient-reported outcomes of patients with advanced renal cell carcinoma treated with nivolumab plus ipilimumab versus sunitinib (CheckMate 214): a randomised, phase 3 trial. Lancet Oncol. 2019;20(2):297-310. https://doi. org/10.1016/S1470-2045(18)30778-2.

20. Wilky BA, Trucco MM, Subhawong TK, Florou V, Park W, Kwon D, et al. Axitinib plus pembrolizumab in patients with advanced sarcomas including alveolar soft-part sarcoma: a single-Centre, single-arm, phase 2 trial. Lancet Oncol. 2019;20(6):837-48. https://doi.org/10.1016/S1470-2045(19)30153-6.

21. Tsao MS, Kerr KM, Kockx M, Beasley MB, Borczuk AC, Botling J, et al. PD-L1 immunohistochemistry comparability study in real-life clinical samples: results of blueprint phase 2 project. J Thorac Oncol. 2018;13(9):1302-11. https://doi.org/10.1016/j.jtho.2018.05.013.

\section{Publisher's Note}

Springer Nature remains neutral with regard to jurisdictional claims in published maps and institutional affiliations. 